\title{
BMJ Open Comparable mRNA expression of inflammatory markers but lower claudin-1 mRNA levels in foreskin tissue of HSV-2 seropositive versus seronegative asymptomatic Kenyan young men
}

\author{
Maria Röhl, ${ }^{1}$ Annelie Tjernlund, ${ }^{1}$ Supriya D Mehta, ${ }^{2}$ Pernilla Pettersson, ${ }^{1}$ \\ Robert C Bailey, ${ }^{2}$ Kristina Broliden ${ }^{1}$
}

To cite: Röhl M, Tjernlund A, Mehta SD, et al. Comparable mRNA expression of inflammatory markers but lower claudin-1 mRNA levels in foreskin tissue of HSV-2 seropositive versus seronegative asymptomatic Kenyan young men. BMJ Open 2015;5:e006627. doi:10.1136/bmjopen-2014006627

- Prepublication history for this paper is available online. To view these files please visit the journal online (http://dx.doi.org/10.1136/ bmjopen-2014-006627).

Received 22 October 2014 Revised 15 January 2015 Accepted 18 January 2015

CrossMark

For numbered affiliations see end of article.

Correspondence to Professor Kristina Broliden; kristina.broliden@karolinska.se

\section{ABSTRACT}

Objectives: Skin biopsies from local sites of herpes simplex virus 2 (HSV-2)-induced ulcers can show infiltrates of inflammatory cells several months after macroscopic healing. We hypothesise that foreskin tissue samples of asymptomatic HSV-2 seropositive men had remaining signs of inflammation at the molecular level. Even in the absence of clinical lesions, genital inflammation may contribute to increased HIV susceptibility on sexual exposure to the virus.

Setting: Foreskin tissue samples were collected from men undergoing elective circumcision in Kisumu, Kenya.

Participants: The foreskin tissue samples $(n=86)$ were stratified into study groups based on HSV-2 serology and assessed for mRNA expression of inflammatory markers. Markers of interest were further assessed by immunohistochemical staining within the tissue samples.

Results: The two study groups had comparable levels of all molecular markers (CD3, CD4, CD8, CD69, CCR5, HLA-DR, Langerin, DC-SIGN, Mannose Receptor 1 , IL-1, IL-6, TNF- $\alpha, \beta 7$, IgA, IFN- $\alpha$, CCL5, E-cadherin, Z0-1 and occludin), except for lower mRNA levels of the epithelial junction protein claudin-1 in the HSV-2 seropositive group $(p=0.008)$. Although mRNA levels of claudin-1 were lower in HSV-2 seropositive individuals, the corresponding protein could be visualised in the foreskin epithelium of all samples tested.

Conclusions: Whereas no general inflammation was demonstrated in the foreskin of asymptomatic HSV-2 seropositive individuals, a decreased expression of claudin-1 indicates a less robust genital epithelial barrier. An intact epithelial barrier is essential for blocking mucosal entry of genital infections, including HIV.

\section{INTRODUCTION}

Herpes simplex virus 2 (HSV-2) is one of the most common sexually transmitted infections

\section{Strengths and limitations of this study}

- The present study is straightforward demonstrating mRNA levels of several markers of inflammation and epithelial barrier integrity from foreskins of herpes simplex virus type 2 (HSV-2) seropositive and seronegative young men undergoing elective circumcision.

- Measuring mRNA transcripts from the tissue samples does not reveal the cell source of the markers, neither does it allow quantification of the markers at the protein level.

- By assessing tissue samples from the time of circumcision, the study can only be crosssectional and data is not available with regard to history of recurrent HSV-2 local lesions.

- The study cannot define whether lower Claudin-1 mRNA levels indicate HSV-2 infection or if HSV-2 infection causes reduced Claudin-1 mRNA levels.

(STIs) of the genital skin and mucosa. During primary infection, HSV-2 infects genital epithelial cells and is then transported via sensory nerves to the sacral root ganglion, where lifelong latency is established. Intermittent genital shedding of HSV-2 in the immunocompetent person is frequent, with or without symptoms. ${ }^{1}{ }^{2}$ In situ analysis of skin biopsies from HSV-2 lesions have revealed cellular infiltrates consisting of inflammatory cells that persisted for months after healing, even with daily antiviral therapy. ${ }^{3}{ }^{4}$ Genital skin lesions and activation of the mucosal immune response at time of local reactivation increase the risk of sexual transmission of the virus, as well as the risk of acquiring other genital infections, 
including HIV. Although daily antiviral therapy against HSV-2 reduces viral replication and visible lesions, the persistent mucosal inflammation of the human skin samples, including upregulation of the HIV coreceptors DC-SIGN and CCR5, may partly explain the inability of the therapy to reduce acquisition of HIV. ${ }^{35}$

Disruption of the male foreskin epithelial barrier is an important risk factor for viral entry during sexual intercourse. The foreskin mucosa is, thus, a highly relevant site for investigating viral transmission, especially in the context of a representative study population of young men living in an HIV endemic region. The male foreskin consists of a keratinised stratified epithelial layer and the epithelial cells express a number of tight junction and adhesion proteins that together form a functional barrier against invading pathogens. ${ }^{6-8}$ This barrier function can easily be disrupted during mucosal inflammation. In human skin, the tight junction complex has three major transmembrane proteins identified as claudin, occludin and junctional adhesion molecules. E-cadherin is a major adhesion protein and is expressed on the basal side of the tight junction protein complex. Among these protein families, claudin-1, occludin and E-cadherin have been identified in foreskin keratinocytes and foreskin tissue. ${ }^{6}{ }^{79}$ Pathogens often use these specific epithelial intercellular junction proteins as a means of entry. In human skin, HSV infects keratinocytes through a complex interaction involving the protein nectin- $1 .^{10}$ Reduction of the expression of another junction protein, claudin-1, is associated with enhanced susceptibility to HSV-1 infection in patients with atopic dermatitis. ${ }^{11}$

HIV can be sexually transmitted, and the male and female genital tract mucosa is an important site of viral entry. Once HIV has crossed the genital epithelial barrier the virus mainly replicates in submucosal CD4+ $\mathrm{T}$ cells and the infection is then further spread to regional lymphnodes and the systemic circulation. ${ }^{12}$ Thus, an inflammatory milieu, defined by increased expression of specific chemokines and other proinflammatory molecules, results in recruitment of $\mathrm{CD} 4+\mathrm{T}$ cells and an increased risk of systemic HIV infection. We here hypothesize that HSV-2 seropositivity is associated with genital mucosal inflammation and a disrupted epithelial barrier, even in asymptomatic individuals. This may theoretically contribute to the higher susceptibility of HIV infection that has been observed in HSV-2 seropositive individuals.

\section{MATERIAL AND METHODS Cohort description}

Foreskin tissues were obtained from a randomised, placebo-controlled clinical trial conducted in Kisumu, Kenya, through the Universities of Nairobi, Illinois and Manitoba Collaborative Research Project. Men aged 18 to 24 years were recruited into an unblinded trial with two arms: the circumcision arm and the delayed circumcision (control) arm to examine whether male circumcision reduced HIV incidence. ${ }^{13}$ For the present study, samples were randomly selected before circumcision and stratified by HSV-2 serological status. Equal numbers of specimens were randomly selected from each stratum and matched for age (18-20 and 21-24 years). The STD screening included a history of discharge and previously diagnosed sexually transmitted infections (STIs), a clinical examination 2 weeks before and at the time of circumcision, as well as laboratory analysis of Neisseria gonorrhoeae, Chlamydia trachomatis, Trichomonas vaginalis, Haemophilus ducreyi (chancroid) and Treponema pallidum (syphilis). ${ }^{14}$ Serum specimens were tested for HSV-2 antibody (Kalon HSV-2 IgG ELISA, Kalon Biological Limited, Aldershot, UK), using the manufacturer's recommended cut-off. ${ }^{14}$

\section{PCR analysis of mRNA expression}

The foreskin tissues were processed immediately in a laboratory at the study clinic by making two horizontal cuts $4 \mathrm{~mm}$ apart and approximately $1.0 \mathrm{~cm}$ from distal end of intact foreskin oriented with the frenulum up centre. The inner and outer mucosa were not specifically marked at the time of processing. The tissue biopsies were immediately embedded in optimal cutting temperature (OCT) and snap frozen following surgery and dissection, and were subsequently stored at $-80^{\circ} \mathrm{C}$. At time of analysis, the OCT was discarded and the biopsies were disrupted in lysis buffer by using a mechanical rotor. RNA was extracted according to the manufacturer's protocol (RNeasy, QIAgen; Hilden, Germany) and converted in equal dilutions to cDNA in a single reverse transcriptase reaction using superscript reverse transcriptase (Invitrogen, Carlsbad, California, USA) and random hexanucleotide primers (Roche, Basel, Switzerland). DNA was stored at $-20^{\circ} \mathrm{C}$. Amplification of the cDNA of CD3, CD4, CD8, CD69, CCR5, HLA-DR, Langerin (CD207), DC-SIGN (CD209), Mannose Receptor 1(MRC1), IL-1, IL-6, TNF- $\alpha$, $\beta 7$, IgA, IFN- $\alpha$, CCL5, E-cadherin, ZO-1, occludin and claudin-1 was performed by using the ABI PRISM 7700 sequence detection system and commercial FAM dye-labelled TaqMan MGB probes and primers (Applied Biosystems, Foster City, California, USA). Each sample and control was run in duplicates. Relative quantity of target cDNA was computed by using the comparative threshold $(\mathrm{Ct})$ method. Ct values for target cDNA were normalised to ubiquitin C (UBC) by using the normalised expression ratio $2^{-\mathrm{dCT}}$; thus amounts of mRNA expression are described as a relative quantity to $\mathrm{UBC}(\mathrm{RQ}, \mathrm{UBC}=1)$.

\section{Immunofluorescent staining}

Immunofluorescent staining was performed on $6 \mu \mathrm{m}$ thick sections of the cryopreserved foreskin biopsies. The sections were fixed in ethanol and acetone. Thereafter, the tissue sections were blocked in phosphate-buffered saline (PBS) with $0.1 \%$ saponin and $0.1 \%$ BSA-C (Aurion, Wageningen, the Netherlands), followed by an overnight incubation at $+4^{\circ} \mathrm{C}$ with primary antibodies: 
the selected mouse IgG monoclonal antibodies detecting human E-cadherin (kindly provided by Dr KJ Green and Dr T Hope, Northwestern University Feinberg School of Medicine, Chicago, Illinois, USA), rabbit-anti-ZO-1 (polyclonal, Invitrogen, Life Technologies Europe BV, Stockholm, Sweden), mouse-anti-occludin (clone OC3F10, Invitrogen), and rabbit-anti-Claudin-1, (polyclonal, Abcam). Thereafter the tissue sections were washed and blocked in PBS containing 0.1\% Saponin, 0.1\% BSA-C and $2 \%$ normal donkey serum for $30 \mathrm{~min}$ at room temperature. This was followed by an $1 \mathrm{~h}$ incubation with either Alexa Fluor-488 conjugated donkey anti-mouse IgG antibody or Alexa fluor-594 donkey-anti-rabbit IgG antibody (Invitrogen/Molecular probes). The sections were washed and the nuclei were stained with DAPI ( $0.75 \mu \mathrm{M} ; 4^{\prime}, 6$-Diamidino-2-Phenylindole, Dihydrochloride, Invitrogen) for $10 \mathrm{~min}$. The tissue sections were mounted in SlowFade Gold Antifade Reagents (Molecular Probes). Negative controls consisted of incubations in the presence of only the secondary antibody.

\section{Statistical analysis}

Characteristics of HSV-2 seronegative and seropositive participants were compared by Pearson $\chi^{2}$ test and with Fisher's exact test when cell sizes were less than 5 . Amounts of expressed mRNA were analysed by unpaired two-tailed Mann-Whitney test. All analyses were performed with Stata/SE V.11.0 software (Stata Corp,
College Station, Texas, USA) and GraphPad Prism 4.00 (GraphPad Software, Inc, La Jolla, California, USA).

\section{RESULTS}

\section{Study population}

Foreskin tissues were obtained from HSV-2 seronegative $(n=43)$ and HSV-2 seropositive $(n=43)$ men undergoing elective circumcision. According to the inclusion criteria, the men had no clinical or laboratory signs of genital ulcers at time of circumcision. Data is not available regarding HSV-1 serostatus, time of primary HSV-2 infection, possible recurrences or location of HSV-2 lesions. Data on sociodemographic, behavioural risks, and STIs for the two study groups were statistically similar, except for educational attainment and number of sex partners which were higher in the HSV-2 seropositive group ( $\mathrm{p}=0.019$ and $\mathrm{p}=0.007$, respectively; table 1 ).

\section{Selected immunological markers were not upregulated in HSV-2 seropositive men}

The overall immune activation status of the foreskin tissue samples was assessed by mRNA expression of a panel of molecules and phenotypic markers. The mRNA expression levels of the cytokines and immunoregulatory receptors IL-1, IL-6, TNF- $\alpha$, IFN- $\alpha$, CCL5, CCR5, HLA-DR and $\beta 7$ as well as of IgA were similar in the study groups $(p=>0.05)$. Likewise, the mRNA expression levels of the

Table 1 Distribution of sociodemographic, behavioural risks and STI outcomes and syndromes by HSV-2 serostatus among uncircumcised men at baseline

\begin{tabular}{|c|c|c|c|}
\hline Variable & $\begin{array}{l}\text { HSV-2 seronegative } \\
\mathrm{N}=43 \\
\mathrm{n}(\%)\end{array}$ & $\begin{array}{l}\text { HSV-2 seropositive } \\
\mathrm{N}=43 \\
\mathrm{n}(\%)\end{array}$ & $\chi^{2}$ p Value* \\
\hline Age group in years & & & 1.000 \\
\hline $18-20$ & $16(37.2)$ & $16(37.2)$ & \\
\hline $21-24$ & $27(62.8)$ & $27(62.8)$ & \\
\hline Educational attainment & & & 0.019 \\
\hline None, primary 1-8 & $10(23.3)$ & $17(39.5)$ & \\
\hline Secondary 1-3 & 7 (16.3) & $13(30.2)$ & \\
\hline Secondary 4, postsecondary & $26(60.5)$ & $13(30.2)$ & \\
\hline Employment status & & & 0.062 \\
\hline Unemployed & $4(9.3)$ & $5(11.6)$ & \\
\hline Self-employed & $12(27.9)$ & $22(51.2)$ & \\
\hline Salaried & $27(62.8)$ & $16(37.2)$ & \\
\hline Married or cohabiting & & & 0.202 \\
\hline No & $42(97.7)$ & $38(88.4)$ & \\
\hline Yes & $1(2.3)$ & $5(11.6)$ & \\
\hline Number of sex partners, past 30 days & & & 0.007 \\
\hline 0 & $22(51.2)$ & $15(34.9)$ & \\
\hline 1 & $21(48.8)$ & $20(46.5)$ & \\
\hline 2 or more & $0(0.0)$ & 8 (18.6) & \\
\hline Frequency of condom use, past 6 months & & & 0.154 \\
\hline Never & $11(25.6)$ & $14(32.6)$ & \\
\hline Sometimes & $21(48.8)$ & $25(58.1)$ & \\
\hline Always & $11(25.6)$ & $4(9.3)$ & \\
\hline Gonorrhea or chlamydia infection at baseline & $1(2.3)$ & $3(7.0)$ & 0.616 \\
\hline
\end{tabular}

${ }^{*}$ Fisher's exact test was used when cell size $n<5$.

HSV 2, herpes simplex virus 2; STI, sexually transmitted infections. 
phenotypic cellular markers CD4, CD3 and CD8, the activation marker CD69, the CLRs Langerin, DC-SIGN and mannose receptor did not differ significantly between the study groups ( $p>0.05$; data not shown).

\section{Lower expression of claudin-1 in the foreskin of HSV-2 seropositive men}

The mRNA expression of the epithelial junction molecules E-cadherin, ZO-1, claudin-1 and occludin were further compared between the two study groups. The HSV-2 seropositive men had significantly lower mRNA expression of claudin-1 (median: 0.161) than the HSV-2 seronegative men (median: 0.294; $\mathrm{p}=0.008$ ), whereas both groups had similar expression levels of E-cadherin, ZO-1 and occludin ( $p=0.495 ; p=0.253 ; p=0.251$, respectively; figure 1A). The mRNA expression was also compared by age and behavioural risks but no significant differences were seen (data not shown).
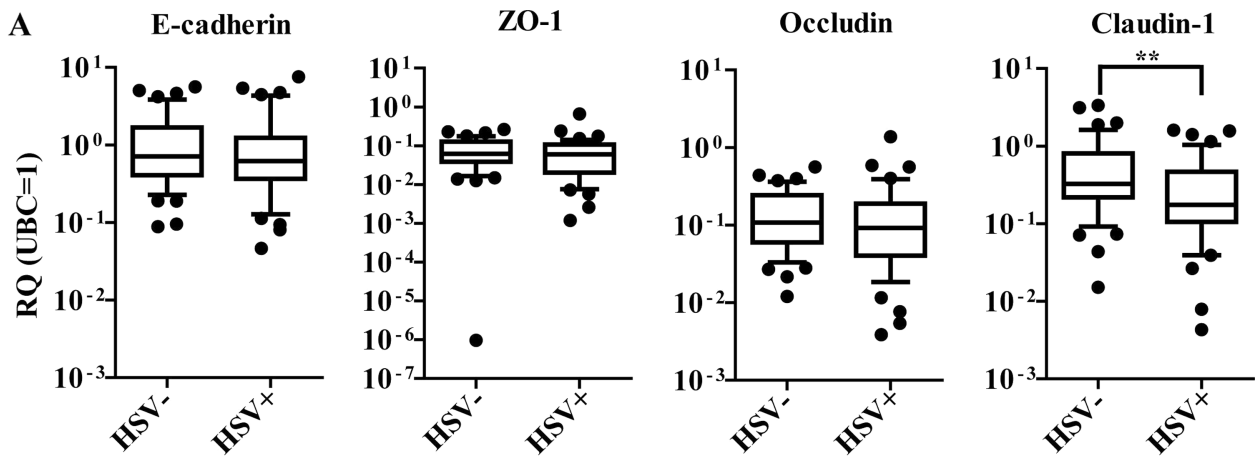

B
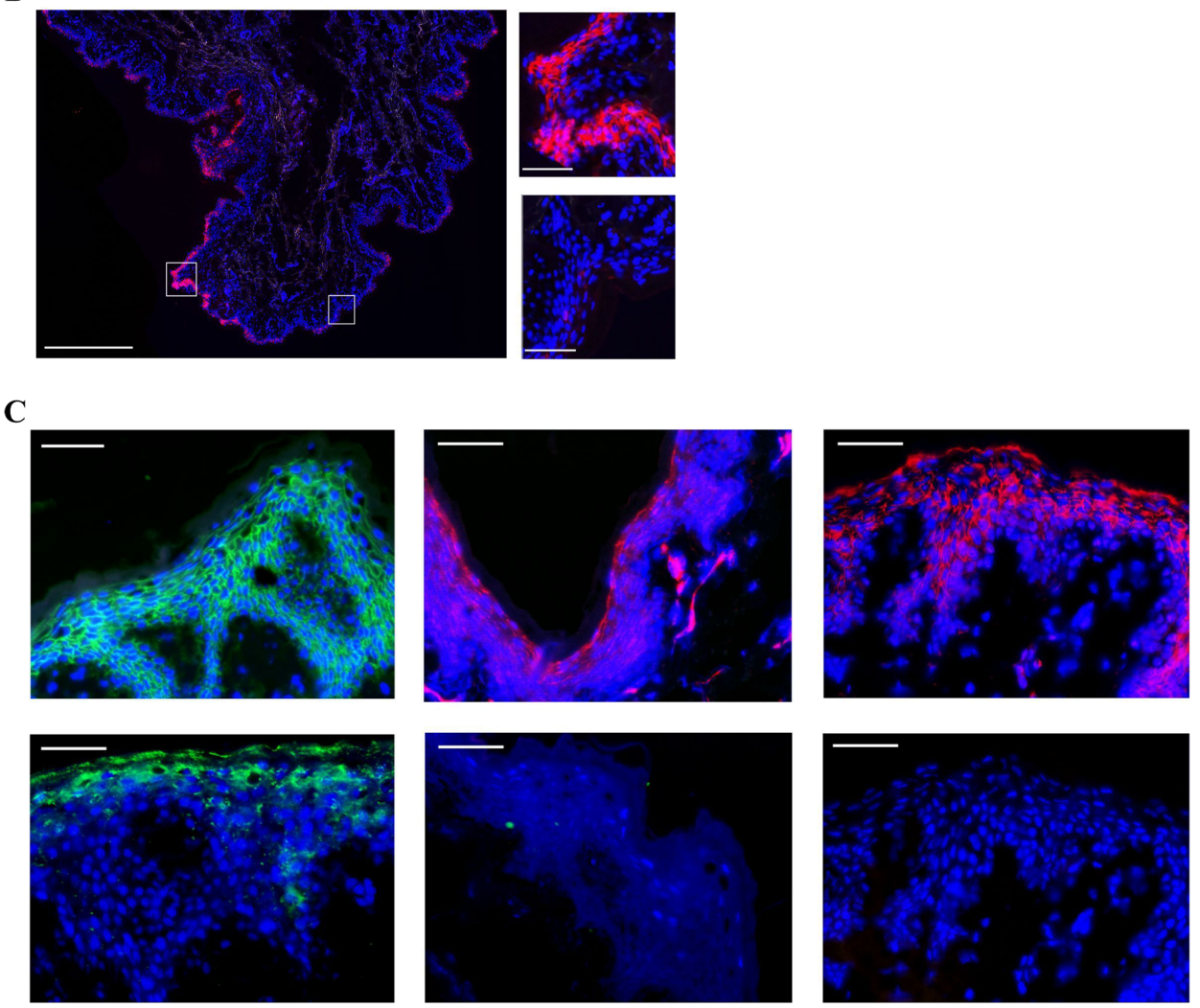

Figure 1 (A) herpes simplex virus 2 (HSV-2) seronegative $(n=43)$ and seropositive men $(n=43)$ were compared for mRNA expression of E-cadherin, ZO-1, occludin and claudin-1 in foreskin tissue samples. The HSV-2 seropositive men had significantly lower expression of claudin-1 $(p=0.008)$, and similar expression of the other three markers $(p>0.05)$. (B) In situ staining of a foreskin tissue section stained for claudin-1 (red) and 4',6-Diamidino-2-Phenylindole, Dihydrochloride, Invitrogen (DAPI; blue) demonstrating the uneven distribution of claudin in the epithelial layer of the foreskin. The figure is a representative image of the sample collection. The images to the right are magnifications of the marked area in the picture to the left. The left image was collected with a $5 \times$ objective (scale bar $=500 \mu \mathrm{m}$ ) and the images to the right with a $40 \times$ objective (scale bar $=50 \mu \mathrm{m})$. $(\mathrm{C}) \mathrm{In}$ situ staining of foreskin tissue sections. Upper row, from left to right: E-cadherin (green), ZO-1 (red) and claudin-1 (red). Lower row, from left to right: Occludin (green), negative control for Alexa-488 (green), negative control for Alexa-594 (red). All tissue sections were stained with DAPI (blue) to visualise cell nucleus. Images were collected with a $40 \times / 1.25$ objective (scale bar=50 $\mu \mathrm{m}$ ). 
Irregular distribution of claudin-1 within the foreskin epithelial layer

The epithelial localisation of claudin-1 was assessed in the foreskin tissues by immunofluorescent staining. Although claudin-1 was clearly present in the foreskin epithelium, the protein was not evenly located in all areas. Some areas of the foreskin surface had only a few stained epithelial layers whereas other areas were thicker (figure 1B). In the comparison, the other junction proteins E-cadherin, ZO-1 and occludin also had individual patterns of expression and were unevenly distributed throughout the epithelium (figure 1C).

\section{DISCUSSION}

We found that the mRNA expression of a panel of inflammatory markers was not associated with HSV-2 serostatus as assessed in male foreskin tissue of asymptomatic Kenyan young men. However, the mRNA expression of claudin-1 was significantly lower in the samples of HSV-2 seropositive than HSV-2 seronegative men. The foreskin tissues were collected during a randomised clinical trial of circumcision and the samples had been stratified for HSV-2 serostatus and matched by age of participant. The study individuals had, according to the enrolment criteria, not had any visible or laboratory confirmed genital lesions prior to sample collection. Subclinical and clinical inflammation is usually characterised by increased expression of cytokines, influx of inflammatory cells and partial disruption of the epithelial barrier as defined by downregulation of epithelial junction proteins. These parameters were, therefore, assessed at the molecular level by measurement of mRNA expression with a panel of such immune markers.

Immunofluorescent staining of the epithelial junction proteins showed the localisation of the proteins E-cadherin, ZO-1, claudin-1 and occludin in the foreskin tissue samples. The distribution over the entire epithelial surface of the samples was irregular, with areas of thick surface interspersed with thin, positively stained cell layers as well as differences in staining intensity. It was, therefore, not possible to standardise quantification at the protein level and hence, we relied on mRNA expression as our read out. The correlation between mRNA and protein expression of these specific epithelial markers has also been evaluated in a study of virus infection of human epithelial cells showing comparable expression levels. ${ }^{15}$

Epithelial junction proteins are highly dynamic and paracellular permeability can be affected by various virus infections, including rhinovirus, acute respiratory coronavirus, influenza, West Nile and ebola viruses. ${ }^{15-19}$ In the case of West Nile virus, the viral capsid degrades claudin proteins, ${ }^{18}$ and infection of epithelial and endothelial cells causes endocytosis of a subset of tight junction proteins, including claudin- $1 .{ }^{20}$ Claudin-1 is also involved in dengue virus and hepatitis $\mathrm{C}$ virus entry. ${ }^{21}{ }^{22}$ Genetic variants of CLDN1 may further alter susceptibility to $\mathrm{HCV}$ infection $^{23}$ and contribute to atopic dermatitis. ${ }^{11}$ Since reductions in claudin-1 expression can enhance susceptibility to HSV-1 infections in atopic dermatitis, ${ }^{11}$ this implicates tight junctions as a critical barrier structure important in containing the spread of epidermal HSV-1 infections. Thus, the lower claudin-1 mRNA expression seen in the HSV-2 seropositive group in the present study is reminiscent of the claudin-1 reduction and enhanced susceptibility to HSV-1 as described above. Altered expression levels of claudin-1 may also affect susceptibility to other genital infections since it represents a more fragile epithelial barrier status. However, this could not be assessed in the present study since men with symptomatic or laboratory-confirmed genital infections were not eligible for circumcision.

The HSV-2 seropositivity in our study is likely to represent previous HSV-2 lesions located on the foreskin in the majority of the participants. ${ }^{24}$ Even though we could not detect persisting inflammation in the foreskin tissue, as defined by mRNA expression of a number of immune markers, this does not exclude effects on additional inflammatory cytokines, such as interferons $\beta$ and $\gamma$, or transforming growth factor- $\beta$. Neither do the results exclude HSV-2 associated longstanding effects on other immune responses as previously described. ${ }^{3425} \mathrm{In}$ future longitudinal studies we plan to evaluate whether claudin-1 is persistently downregulated following HSV-2 lesions. The present cross-sectional study format does not allow assessment of the temporal role of HSV-2 for the reduction of claudin- 1 mRNA expression. The study participants may also have had a prior (genetic or induced) defect in claudin-1 expression which increased their HSV-2 susceptibility. The men in the present study were sexually active and living in an HIV endemic geographical area and we have thus demonstrated that their HSV-2 serostatus was not associated with general mucosal inflammation at the foreskin tissue level. The association between HSV-2 seropositivity and a lower claudin-1 mRNA expression warrants further studies, since a specific defect of the genital mucosal barrier in a highly vulnerable and relevant population could be of significance. A less robust genital epithelial barrier in HSV-2 seropositive men would be a pathogenic factor by facilitating entrance of STIs, including HIV. Topical compounds strengthening the epithelial layer are an interesting prophylactic approach to lower sexual transmission and acquisition of genital infections.

\section{Author affiliations}

${ }^{1}$ Unit of Infectious Diseases, Department of Medicine Solna, Karolinska University Hospital, Center for Molecular Medicine, Karolinska Institutet, Stockholm, Sweden

${ }^{2}$ Division of Epidemiology, School of Public Health, University of Illinois at Chicago, Chicago, Illinois, USA

Acknowledgements The authors thank the study staff, the study participants and Dr S Moses for valuable contributions to the study.

Contributors MR, SDM and PP have contributed to the acquisition, analysis and interpretation of data for the work. AT, RCB and KB have contributed to the conception and design of the work as well as analysis and interpretation of data for the work. All authors have critically drafted or revised the work for important intellectual content, finally approved the version to be published. 
Funding This work was supported by the Swedish Research Council [to KB], the Swedish International Development Cooperation Agency, SIDA/SAREC (to $\mathrm{KB}$ ), and from the National Institute of Allergy and Infectious Diseases (NIAID), Division of AIDS, National Institute of Health (NIH) (to RCB, Al50440). RCB was partially supported by the Chicago Developmental Centre for AIDS Research (D-CFAR), an NIH funded programme (P30 AI 082151).

Competing interests None.

Ethics approval University of Chicago, University of Manitoba, Karolinska Institutet, University of Nairobi.

Provenance and peer review Not commissioned; externally peer reviewed.

Data sharing statement No additional data are available.

Open Access This is an Open Access article distributed in accordance with the Creative Commons Attribution Non Commercial (CC BY-NC 4.0) license, which permits others to distribute, remix, adapt, build upon this work noncommercially, and license their derivative works on different terms, provided the original work is properly cited and the use is non-commercial. See: http:// creativecommons.org/licenses/by-nc/4.0/

\section{REFERENCES}

1. Wald A, Corey L, Cone R, et al. Frequent genital herpes simplex virus 2 shedding in immunocompetent women. Effect of acyclovir treatment. J Clin Invest 1997;99:1092-7.

2. Wald A, Zeh J, Selke S, et al. Virologic characteristics of subclinical and symptomatic genital herpes infections. $N$ Engl J Med 1995;333:770-5.

3. Zhu J, Hladik F, Woodward A, et al. Persistence of HIV-1 receptor-positive cells after $\mathrm{HSV}-2$ reactivation is a potential mechanism for increased HIV-1 acquisition. Nature Med 2009;15:886-92.

4. Zhu J, Peng T, Johnston $\mathrm{C}$, et al. Immunosurveillance by CD8aa+ skin-resident $T$ cells in human herpes virus infection. Nature 2013;497:494-7.

5. Prodger JL, Gray R, Kigozi G, et al. Impact of asymptomatic herpes simplex virus-2 infection on $T$ cell phenotype and function in the foreskin. AIDS 2012;26:1319-22.

6. Tebbe B, Mankertz J, Schwarz S, et al. Tight junction proteins: a novel class of integral membrane proteins. Arch Dermatol Res 2002:294:14-18.

7. Dinh $\mathrm{MH}$, Okocha EA, Koons A, et al. Expression of structural proteins in human female and male genital epithelia and implications for sexually transmitted infections. Biol Reprod 2012;86:1-6.

8. Hirbod T, Bailey RC, Agot K, et al. Abundant expresison of HIV target cells and C-type lectin receptors in the foreskin tissue of young Kenyan men. Am J Pathol 2010;176:2798-805.
9. Lemos MP, Lama JR, Karuna ST, et al. The inner foreskin of healthy males at risk of HIV infection harbors epithelial CD4+ CCR5+ cells and has features of an inflamed epidermal barrier. PLOS ONE 2014;9:e108954.

10. Geraghty RJ, Krummenacher C, Cohen GH, et al. Entry of alphaherpesviruses mediated by poliovirus receptor-related protein 1 and poliovirus receptor. Science 1998;280:1618-20.

11. De Benedetto A, Slifka MK, Rafaels NM, et al. Reductions in claudin-1 may enhance susceptibility to herpes simplex virus 1 infections in atopic dermatitis. J Allergy Clin Immunol 2011;128:242-6.

12. Haase AT. Targeting early infection to prevent HIV-1 mucosal transmission. Nature 2010;464:217-23.

13. Bailey RC, Moses S, Parker CB, et al. Male circumcision for HIV prevention in young men in Kisumu, Kenya: a randomised controlled trial. Lancet 2007;369:643-56.

14. Mehta SD, Moses S, Agot K, et al. Adult male circumcision does not reduce the risk of incident Neisseria gonorrhoeae, Chlamydia trachomatis, or Trichomonas vaginalis infection: results from a randomized, controlled trial in Kenya. J Infect Dis 2009;200:370-8.

15. Yeo NK, Jang YJ. Rhinovirus infection-induced alteration of tight junction and adherens junction components in human nasal epithelial cells. Laryngoscope 2010;120:346-52

16. Teoh KT, Siu YL, Chan WL, et al. The SARS coronavirus E protein interacts with PALS1 and alters tight junction formation and epithelial morphogenesis. Mol Biol Cell 2010;21:3838-52.

17. Golebiewski L, Liu H, Javier RT, et al. The avian influenza virus NS1 ESEV PDZ binding motif assocaites with Dlg1 and Scribble to disrupt cellular tight junctions. J Virol 2011;85:10639-48.

18. Medigeshi GR, Hirsch AJ, Brien JD, et al. West Nile virus capsid degradation of claudin proteins disrupts epithelial barrier function. J Virol 2009;83:6125-34.

19. Wahl-Jensen VM, Afanasieva TA, Seebach J, et al. Effects of Ebola virus glycoproteins on endothelial cell activation and barrier function. J Virol 2005;79:10442-50.

20. Xu Z, Waeckerlin R, Urbanowski MD, et al. West Nile virus infection causes endocytosis of a specific subset of tight junction membrane proteins. PLOS ONE 2012:7:e37886.

21. Gao F, Duan X, Lu X, et al. Novel binding between pre-membrane protein and claudin-1 is required for efficient dengue virus entry. Biochem Biophys Re Comm 2009;391:952-7.

22. Evans MJ, von Hahn T, Tscherne DM, et al. Claudin-1 is a hepatitis $\mathrm{C}$ virus co-receptor required for a late step in entry. Nature 2007;446:801-5.

23. Bekker V, Chanock SJ, Yeager M, et al. Genetic variation in CLDN1 and susceptibility to hepatitis $C$ virus infection. $J$ Viral Hepatitis 2010;17:192-200.

24. Mehta SD, Moses S, Parker CB, et al. Circumcision status and incident HSV-2 infection, genital ulcer disease, and HIV infection. AIDS 2012;26:1141-9.

25. Zhu J, Koelle DM, Cao J, et al. Virus-specific CD8+T cells accumulate near sensory nerve endings in genital skin during subclinical HSV-2 reactivation. J Exp Immunol 2007;204:593-603. 